\title{
Diagnostic and Management Problems of Chylous Effusion in A Patient with Newly-Diagnosed Tuberculosis
}

\author{
Dicky Febrianto ${ }^{*}$, Usman Hadi ${ }^{2}$ \\ ${ }^{1}$ Faculty of Medicine, Universitas Airlangga - Dr. Soetomo General Academic Hospital, Surabaya, Indonesia \\ ${ }^{2}$ Department of Internal Medicine, Faculty of Medicine - Dr. Soetomo General Academic Hospital, Surabaya, Indonesia
}

\begin{abstract}
A B S T RA C T
Tuberculosis (TB) remains a major cause of morbidity and mortality globally. Although TB most commonly affects the lungs, any organ or tissue can be involved. Extra-pulmonary forms of TB are commonly unrecognized or late diagnosed. Chylous effusion, i.e. chylothorax and chylous ascites, which is characterized by the presence of chyle in the pleural and peritoneal cavities, is an uncommon manifestation of extra-pulmonary TB. A 22-year-old male, referred to Dr. Soetomo Hospital with complaints of dyspnea, fever, and abdominal distension. Chest X-ray showed pleural effusion. Analysis of fluid obtained from thoracentesis and paracentesis showed chylothorax and chylous ascites. M. tuberculosis had been found in sputum smear examination. ADA (adenosine deaminase) test was performed on ascites fluid and a positive result was obtained. Chylous effusion in this patient were concluded to be related to TB. Patient was then treated with anti-TB drugs and somatostatin. Chylothorax and chylous ascites improved after treatment with somatostatin for 1 week. Administration of anti-TB drugs was planned to be continued for 9 months. The most common causes of non-traumatic chylous effusion in developing countries are infection of TB and filariasis. Chylous effusion is caused by obstruction or disruption of the lymphatic system. ADA test is a new biomedical method that begins to expand its use in body fluids to diagnose extra-pulmonary TB. Fasting, together with total parenteral nutrition, can decrease the lymph flow and balance metabolic impairment. Somatostatin has been used in the treatment of chylous effusion as it diminishes peristalsis and intestinal absorption of fats as well as decreases portal pressure.
\end{abstract}

Keywords: Extra-pulmonary TB, Chylous effusion, Chylothorax, Chylous ascites, ADA test

Correspondence: Dicky Febrianto

E-mail: dicky.febrianto-2014@fk.unair.ac.id

Article history: •Received 10 December $2020 \bullet$ Received in revised form 29 January $2021 \bullet$ Accepted 5 February 2021

\section{INTRODUCTION}

Tuberculosis (TB) is one of the main causes of morbidity and mortality globally and it kills about two million people annually (Afrasiabian et al.,2013). In 2001, the World Health Organization (WHO) reported 2.4 million cases and approximately 2 billion people worldwide have latent TB infection. During 2008, an estimated 9.4 million new TB cases were diagnosed, with most cases living in Africa and Asia (Tseng et al., 2014).

TB is a granulomatous disease caused by Mycobacterium tuberculosis. TB primarily affects the lungs, but all organs may be affected. Extra-pulmonary forms of TB pose a big public health problem, because unlike pulmonary forms that lead patients quickly at health centers, they are commonly unrecognized or late diagnosed, which delay the treatment. This delay in diagnosis is not only due to the delay in the examination, but also due to the diagnostic difficulties related to extra-pulmonary TB (Tchaou et al., 2016).

Chylous effusion, i.e. chylothorax and chylous ascites, which is characterized by the presence of chylous fluid in the pleural and peritoneal cavities, is an uncommon manifestation of extra-pulmonary TB (Kim et al., 2014). Diagnosis of extrapulmonary TB often requires invasive procedures.
There is need of precise and faster diagnosis for patient with extra-pulmonary TB. Accordingly, there develops tests based on biochemical response of body towards TB infection. Adenosine deaminase (ADA) test is one of biochemical test for TB (Stevanovic et al., 2011; Ninghot et al., 2017).

The management of chylous effusion is still a challenge. Therapeutic thoracentesis is the initial step in large chylothorax that cause respiratory distress. The cornerstone of therapy revolves around correcting the underlying disease and supportive strategies. Fasting, total parenteral nutrition, and somatostatin or octreotide are the supportive therapies for chylous effusion (Nair et al., 2007; Yang et al., 2013; Lizaola et al., 2017).

Here we reported a problematic case of young man consulted to our department with the complaints of dyspnea and abdominal discomfort due to chylothorax and chylous ascites. M. tuberculosis had been found in sputum smear examination, so that we concluded that chylothorax was related with pulmonary TB. Meanwhile, the cause of chylous ascites are investigated further by radiological and laboratory examination. The difficulty of diagnosing and managing chylous effusion as the extra-pulmonary TB makes this case interesting for further discussion. 


\section{CASE REPORT}

A 22-year-old man, Mr. K, unmarried, having his address at Mojokerto, East Java, referred by emergency room of Mojokerto Hospital with a diagnosis of fluidopneumothorax, coming to the emergency room of Dr. Soetomo Hospital, Surabaya, on January 9th, 2018, with chief complaint of dyspnea since the last month which worsened 2 days before admission. Shortness of breath improved with rest and lying down to the left. Patient also complained of coughing for 5 months, coughing with thick white sputum. Patient got his weight loss since the last 4 months. A history of coughing up blood was denied. Patient complained that his stomach was bloated since the last 4 months.

Physical examination of the patient showed the awareness was compos mentis, GCS of 456, and visual analog scale (VAS) of 2. Vital signs: blood pressure of 130/80 $\mathrm{mmHg}$, pulse rate of 105 times/minute, respiratory rate of 24 times/minute, temperature $37.8^{\circ} \mathrm{C}$, body weight of $50 \mathrm{~kg}$, body height of $160 \mathrm{~cm}$. On physical examination, it was found tachypnea. Breath sound was absent on right side, vocal fremitus and vocal resonance were absent on right side, and percussion was dull on right side. On the examination of abdomen, it was found with positive shifting dullness. On the examination of the extremities, it was obtained edema of both inferior extremities.

Automated blood count demonstrated leucocyte count of 11,640 cell $/ \mathrm{mm}$ with neutrophils of $81.1 \%$, hemoglobin of $13.7 \mathrm{~g} / \mathrm{dL}$, platelet count of $378,000 / \mathrm{mm}^{3}$, blood glucose of $100 \mathrm{mg} / \mathrm{dL}$, BUN of $20 \mathrm{mg} / \mathrm{dL}$, serum creatinine of 0.63 $\mathrm{mg} / \mathrm{dL}$, ALT of $34 \mathrm{U} / \mathrm{L}$, AST of $23 \mathrm{U} / \mathrm{L}$, albumin of 3.07 $\mathrm{g} / \mathrm{dL}$, direct bilirubin of $0.93 \mathrm{mg} / \mathrm{dL}$, total bilirubin of 1.24 $\mathrm{mg} / \mathrm{dL}$, sodium of $133 \mathrm{mmol} / \mathrm{L}$, potassium of $4.5 \mathrm{mmol} / \mathrm{L}$, chloride of $102 \mathrm{mmol} / \mathrm{L}, \mathrm{LDH}$ of 243 (normal: 85-227), PPT of 12.2 seconds (control: 10.3), APTT of 25.0 seconds (control: 27.1), non-reactive rapid test for HIV, and nonreactive HBsAg. Urinalysis showed negative glucose, bilirubin $1+$, ketone $1+$, protein $2+$, negative nitrite, erythrocytes of $25-50$ cells $/ \mathrm{mm}^{3}$, leukocytes of $5-10$ cells $/ \mathrm{mm}^{3}$. Blood gas analysis showed $\mathrm{pH}$ of 7.48; $\mathrm{pCO}_{2}$ of 33.1 ; $\mathrm{pO}_{2}$ of $225 ; \mathrm{HCO}_{3}$ of $25.1 ; \mathrm{BE}$ of $1.4 ; \mathrm{SO}_{2}$ of $99 \%$. Radiological examination of thorax showed fluido-pneumothorax of right lung and pleuropneumonia of left lung.

In the emergency room, patient underwent pleural puncture, with the result of thick and milky white pleural fluid. Patient was admitted to Pulmonology ward, with the initial diagnoses of fluidopneumotorax of right lung, suspected new case of pulmonary TB, ascites, hypoalbuminemia, and edema of inferior extremities. Patient was consulted to Internal Medicine Department regarding the ascites, and a proof puncture of ascites was planned. Patient were also consulted to Cardiology Department regarding inferior extremity edema, however the echocardiographic examination was within normal limits. Patient was planned for chest tube insertion. Patient was administered oxygen via simple mask 6-8 liters/minute, infusion of branched-chain amino acid, intravenous injection of furosemide, spironolactone tablets, and albumin capsules.

On 2nd day of care (January 10th, 2018), patient still complained of dyspnea and abdominal discomfort. Patient underwent chest tube insertion and it was obtained milky white pleural fluid with a production of $400 \mathrm{~mL} / 24$ hours. Analysis of pleural fluid showed $\mathrm{pH}$ of 8 (normal: 3.4-5.0), glucose of 122 (normal: <100), LDH of 120 (normal: 100$190)$, leukocyte count of $187 / \mathrm{mm}$, mononuclear cells of $64.2 \%$, polymorphonuclear cells of $35.8 \%$, total protein of $1.8 \mathrm{~g} / \mathrm{dL}$ (normal 6.4-8.2), total cholesterol of $4 \mathrm{mg} / \mathrm{dL}$ (normal: 0-200), triglycerides of $378 \mathrm{mg} / \mathrm{dL}$ (normal: 30150). Blood lab tests showed hemoglobin of $14.2 \mathrm{~g} / \mathrm{dL}$, leukocytes of $15,680 / \mathrm{mm}^{3}$ with neutrophils of $90.9 \%$, platelet count of $323,000 / \mathrm{mm}^{3}$, albumin albumin of $2.7 \mathrm{~g} / \mathrm{dL}$, total cholesterol of $120 \mathrm{mg} / \mathrm{dL}$, triglyceride of $82 \mathrm{mg} / \mathrm{dL}$, nonreactive anti-HCV, AFP of 2.0 (normal: $<15$ ), BUN of 11 $\mathrm{mg} / \mathrm{dL}$, serum creatinine of $0.7 \mathrm{mg} / \mathrm{dL}$, and CRP of 9.5 (normal: 0-1). Patient was performed a proof puncture of ascitic fluid, and a milky white liquid was obtained. Patient was diagnosed as chylothorax of right lung e.c. suspected new case of TB, ascites, hypoalbuminemia, and inferior limb edema. Patient was fasted, received oxygen mask therapy 6 liters/minute, infusion of $20 \%$ fat emulsion 500 $\mathrm{mL} /$ day, infusion of $10 \%$ dextrose $500 \mathrm{~mL} /$ day, furosemide $20 \mathrm{mg}$ t.i.d. intravenously, ceftriaxone 1 gram b.i.d. intravenously, metronidazole $500 \mathrm{mg}$ t.i.d. intravenously, and spironolactone tablets $100 \mathrm{mg}$ once daily.

On 4th day of care (January 12th, 2018), CT scan of thorax showed pneumonia, bilateral pleural effusions, and chest tube via the right intercostal space III with a distal tip projected as high as vertebra thoracal-1. There was no picture of pneumothorax. CT scan of abdomen showed the presence of ascites, bilateral pleural effusion, and lymph node enlargement in the left and right inguinal paraaortas. The ascitic fluid analysis demonstrated $\mathrm{pH}$ of 8 (3.4-5.0), glucose of $115 \mathrm{mg} / \mathrm{dL}$ (normal: <100), LDH of 50 (normal: 100-190), leukocyte count of $226 / \mathrm{mm}^{3}$, mononuclear cells of $86.3 \%$, polymorphonuclear cells of $13.7 \%$, total protein of $1.4 \mathrm{~g} / \mathrm{dL}$ (normal 6.4-8.2), total cholesterol of $6 \mathrm{mg} / \mathrm{dL}$ (normal: 0-200), and triglyceride of $509 \mathrm{mg} / \mathrm{dL}$ (normal: 30150). Cytology examination of ascitic fluid showed no malignant cells, bacteria, fungi, and smear, and negative results of Gram staining. Patient was diagnosed as chylothorax of right lung e.c. suspected new case of pulmonary TB, chylous ascites, hypoalbuminemia, leukocytosis, and inferior limb edema. Patient was fasted, administered infusion of $20 \%$ fat emulsion $500 \mathrm{~mL} /$ day, infusion of $10 \%$ dextrose $500 \mathrm{~mL} /$ day, and pump infusion of somatostatin $6 \mathrm{mg} / 24$ hours. 
On 7th day of care (January 15th, 2018), patient's complaint of dyspnea had diminished. Production of pleural fluid measured from chest tubes had decreased to less than 50 mL/day. Smear sputum examination showed $2+$ result. GenXpert (nucleic acid amplification) examination showed positive for $M$. tuberculosis bacteria without rifampicin resistance.

Patient's complaints had diminished on the 9th day of care (January 17th, 2018). Abdominal discomfort had improved. The chest tube had been removed. Blood laboratory examination showed procalcitonin of 0.12 (local infection), C3 complement of 129 (normal: 50-120), C4 complement of 25.8 (normal: 20-50), ANA test of 25.91 (indeterminate), lipase of 25 IU/L (normal: 10-150), amylase of 50 IU/L (35118), CEA of $2.5 \mathrm{ng} / \mathrm{mL}$ (normal: <3), and Ca-19.9 of 15 U/mL (normal: 0-37). ADA test for ascitic fluid was $50 \mathrm{IU} / \mathrm{L}$ (normal: <36 IU/L). Thoracic CT scan for evaluation showed no visible picture of pneumothorax, pneumonia, and pleural effusion. Finally, this patient was diagnosed as chylothorax related to new case of pulmonary $\mathrm{TB}$, chylous ascites related to peritoneal TB, and hypoalbuminemia. Patient was begun receiving enteral diet, infusion of branched chain amino acid $500 \mathrm{~mL} / 24$ hours, drinking $700 \mathrm{~mL} /$ day, albumin capsules t.i.d. orally, and category I FDC anti-TB drugs of 3 tablets/day (total rifampicin of $450 \mathrm{mg}$, isoniazid of $225 \mathrm{mg}$, pyrazinamide of $1200 \mathrm{mg}$, ethambutol of $825 \mathrm{mg}$ ). Somatostatin was discontinued after 6 days of administration as the patient's clinical condition improved.

On 11th day of care (January 19th, 2018), complaints of dyspnea and abdominal discomfort had significantly improved. Patient was discharged. Category I anti-TB drugs and albumin capsules were continued. Patient was then followed-up in DOTS Outpatient Clinic to continue the antiTB drugs for 9 months.

\section{DISCUSSION}

Chylous effusion, which consists of chylothorax and chylous ascites, is an uncommon clinical entities characterized by the accumulation of triglyceride-rich chylous fluid in the pleural and peritoneal spaces. The reported incidence of combined occurrence of chylothorax and chylous ascites has varied from $9 \%$ to $55 \%$ of chylous ascites. No differences in sex distribution have been cited. Chylous ascites is uncommon, with an occurrence of one in 20,000 hospital admission (Tchaou et al., 2016; Kim et al., 2014; Wolf, 2018).

Clinical features of chylotorax are typical of any pleural effusion. Dyspnea, cough, and chest discomfort are the main symptoms. Pleuritic chest pain and fever are uncommon because chylous fluid is not irritating to the pleural surface. The severity of symptoms depends on the size of chylothorax. The existence of symptoms such as weight loss, night sweating, hemoptysis, and a previous history of TB, should lead us to perform appropriate test for TB (Nair et al., 2007; Karapolat et al., 2008).
Progressive and painless abdominal distention (81\%) and non-specific pain (14\%) are the most common presenting symptoms in chylous ascites, occurring over a course of weeks to months depending on the underlying cause. Patients who have undergone abdominal or thoracic surgery may present with an acute onset of chylous ascites. Other features include weight loss, anorexia, malaise, steatorrhea, malnutrition, enlarged lymph nodes, fevers, and night sweating. From physical examination, chylous ascites can't be distinguished from ascites in general (Al-Busafi et al.,2014).

\section{Etiology and Pathophysiology of Chylous Effusion}

The causes of chylous effusion can be categorized as traumatic and non-traumatic. The most common traumatic cause is trauma after abdominal surgery. The traumatic causes of chylous effusion differ between developed and developing countries. In developed countries the most common causes are malignancy (hepatoma, lymphoma, angiosarcoma) and hepatic cirrhosis, while the most common causes in developing countries are infectious diseases (TB and filariasis). Other causes are spontaneous bacterial peritonitis, peritoneal dialysis, carcinoid syndrome, congenital disorders, Kaposi sarcoma, superior cava vein thrombosis, mediastinal fibrosis, hypothyroidism, nephrotic syndrome, pancreatitis, ischemic cardiomyopathy, constrictive pericarditis, systemic lupus erythematosus (SLE), and Henoch-Schonlein purpura (Tchaou et al., 2016; Kim et al., 2014; Wolf, 2018).

Infections with mycobacterial species, as M. avium and M. tuberculosis, tend to induce chylous effusion and contribute to $10 \%$ of all cases. Except for mycobacteria, no other bacterial or viral infection cause chylous ascites (Steinemann et al., 2011). TB needs to be suspected as the etiology in cases of chylous ascites, especially in developing countries like Indonesia, where the prevalence of $\mathrm{TB}$ is still high (Barman et al., 2015).

The combined occurrence of chylothorax and chylous ascites is usually caused by obstruction or disruption of the thoracic duct or of one of its main divisions, which is usually the result of malignancy, trauma, or inflammation. Chylous fluid can experience reflux from pleural to peritoneal cavities or vice versa through a diaphragm fistula (Kumae et al., 2013; Cueto-Aguilera et al., 2016). During mycobacterial infections, the activation of $\mathrm{T}$ cell receptors expressing CD4+ $\mathrm{T}$ cells is essential in the formation of granulomas. Granulomas are formed from a complex cascade of systems. Sensitized type I T-helper are attracted to the site of focally aggregated macrophages that contain ingested bacilli in the case of Mycobacterium infections, and the early granuloma organizes into its characteristic structure. The chylous effusion found in TB patient is likely a result of granulomatous infiltration of the lymphatic system, causing obstruction, in the setting of the abrupt restoration of the immune system (Dean et al., 2018). 
Chylous effusion is the pathologic leakage of triglyceridesrich lymphatic fluid into pleural and peritoneal cavity. Because its volume is very large and rich in nutrients, it may lead to malnutrition, dehydration, electrolyte imbalance, and delayed wound healing. Moreover, as chylous effusion contains lymphatic fluid which is rich in lymphocytes and immunoglobulins, severe and long-term chylous leakage may cause hypoimmunity, therefore leading to severe infection or even death because of sepsis (Pan et al., 2016).

In this patient, there was no history of trauma, chest and abdominal CT scan showed no signs of malignancy, and cytological analysis of ascitic fluid did not show any malignant cell. Constrictive pericarditis, one of the causes of chylothorax and chylous ascites, was not obtained at echocardiography examination. In this patient, there was no other risk factors for chylothorax other than TB.

\section{Diagnosis of Chylous Effusion}

History taking can be very helpful in establishing the diagnosis. There is often a delay in diagnosis of TB, especially extra-pulmonary $\mathrm{TB}$, because the symptoms are not specific.

\section{Radiological Examination}

Radiological examination plays an important role in the evaluation of chylous effusion. Chest X-ray with lateral views as well as decubitus views may be helpful in determining the size and location of the chylothorax, however it cannot differ chylothorax from simple pleural effusion (Nair et al., 2007). Lymphangiography is the gold standard diagnostic tool in cases of lymphatic obstruction. Lymphangiography and lymphoscintigraphy are useful in detecting abnormal retroperitoneal nodes, leakage, fistulization, and patency of the thoracic duct. These techniques are also effective for selecting patients for surgery and assessing the effects of treatment. CT scan and MRI are not specific to chylous ascites, however, they are useful in identifying intrathoracal and intraabdominal masses, fluid collections, or lymph nodes. The CT density of chylous ascites resembles that of water and is indistinguishable from simple pleural fluid, urine, bile, bowel secretions, or simple ascites (Lizaola et al., 2017).

In this patient, chest X-ray showed fluidopneumothorax and pleuropneumonia. CT scan abdomen showed ascites, bilateral pleural effusion, and lymph node enlargement in paraaorta as well as in right and left inguinal regions. However, these examination still could not determine the specific cause of fluidopneumothorax and ascites.

\section{Mycobacterial Stain and Culture}

A definitive diagnosis of TB can only be made by culturing M. tuberculosis organisms from a specimen obtained from the patient. However diagnosing extra-pulmonary TB remains challenging because clinical samples obtained from relatively inaccessible sites may be paucibacillary, decreasing the sensitivity of diagnostic tests. Since the conventional smear microscopy has a low sensitivity with a range of $0 \%-$ $40 \%$, negative results cannot exclude the presence of TB. The reported yields of mycobacterial culture vary from $30 \%$ up to $80 \%$, but it usually takes 2 to 8 weeks to receive the results, which is too slow to help treatment decisions (Lee, 2015).

About $10 \%-50 \%$ of extra-pulmonary TB patients have concomitant pulmonary involvement. Therefore, all suspected cases of extra-pulmonary TB should be assessed for concomitant pulmonary TB to determine whether the case is infectious and to assist with diagnosis. Some extrapulmonary TB patients have positive sputum culture results despite normal chest radiography findings (Lee, 2015).

A Ziehl-Neelsen stain can reveal AFB only if the sample contains greater than 10,000 bacilli per mL. Different culture methods, such as Lowenstein-Jensen medium, radiometric (Bactec 12B liquid medium), and non-radiometric (Bactec MGIT 960 system), can be used for confirming diagnosis in the paucibacillary state (Tseng et al., 2014).

In this patient, M. tuberculosis had been found in sputum smear examination. Mycobacterial cultures of sputum, pleural and ascites fluid were not performed due to it took too long time to receive the result, which was too slow to help treatment decisions. Based on the positive result of sputum smear examination, chylothorax in this patient was very likely related to pulmonary TB.

\section{Chylous Effusion Fluid Analysis}

Pleural puncture and abdominal paracentesis are the most important diagnostic tools in evaluating and managing patients with chylous effusion. In contrast to the yellow and transparent appearance of pleural effusion and ascites due to cirrhosis and portal hypertension, chylous fluid typically has a cloudy and turbid appearance. This should be distinguished from pseudochylous effusion, in which the turbid appearance is due to cellular degeneration from infection or malignancy without actually containing high levels of triglycerides. The serum to ascites albumin gradient (SAAG) should be calculated to determine if the ascites is related to portal hypertension or other causes (Al-Busafi et al., 2014).

The diagnosis of chylothorax and chylous ascites is made by analyzing the fluid obtained through pleural puncture and abdominal paracentesis. Among others, usual findings that point towards the identification of this condition are milky white fluid with triglyceride levels greater than $200 \mathrm{mg} / \mathrm{dL}$, although some authors use a cutoff value of $110 \mathrm{mg} / \mathrm{dL}$, and high leukocyte count of mononuclear cell predominance (AlBusafi et al., 2014; Shaik et al., 2014).

In recent years, there has been a great demand for finding new biomedical diagnostic method to diagnose TB quickly 
and accurately. Measuring adenosine deaminase (ADA) activity is a biological method, especially to diagnose extrapulmonary TB through body fluid. ADA is an enzyme in the purine salvage pathway that catalyzes the conversion of adenosine and deoxyadenosine to inosine and deoxyinosine with the release of ammonia. ADA is essential for proliferation and differentiation of lymphoid cells, especially $\mathrm{T}$ cells, and helps in the maturation of monocytes to macrophages. Activity of this enzyme increases in TB patients. In some studies, the level of ADA in sputum and serum was used for diagnosis of $\mathrm{TB}$, and it was monitored during TB treatment (Afrasiabian et al.,2013; Stevanovic et al., 2011).

A TB smear and culture as well as ADA test should be performed in selected cases when TB is suspected. ADA has high sensitivity and specificity in the diagnosis of TB peritonitis (Al-Busafi et al., 2014). The sensitivity and specificity for diagnosing TB have been reported to be $100 \%$ and $97 \%$ respectively, using cut-off values from 36 to 40 IU/L, with the optimal cutoff point of 39 IU/L (Lee, 2015). Other causes of increase in ADA activity include bacterial infections, rheumatic disease, and lymphoproliferative disorders. ADA test is still an effective method to distinguished TB from non-TB through pleural, ascitic, synovial fluid, and cerebrospinal fluid (Stevanovic et al., 2011).

Table 1. Characteristics of Pleural and Ascitic Fluid in Patient

\begin{tabular}{lccc}
\hline & $\begin{array}{c}\text { Normal } \\
\text { Range }^{12}\end{array}$ & \multicolumn{2}{c}{ Findings in Patient } \\
\cline { 3 - 4 } & $0-200 \mathrm{mg} / \mathrm{dL}$ & 4 & Pleural Fluid \\
\hline Total cholesterol & $30-150 \mathrm{mg} / \mathrm{dL}$ & 378 & Ascitic Fluid \\
\hline Triglycerides & $3.4-5.0$ & 8.0 & 509 \\
\hline $\mathrm{pH}$ & $<100$ & 122 & 8.0 \\
\hline Glucose & $100-190$ & 120 & 50 \\
\hline LDH & & 187 & 226 \\
\hline Leukocyte & & $64.2 \%$ & $86.3 \%$ \\
\hline Mononuclear cell & & $35.8 \%$ & $13.7 \%$ \\
\hline Polymorphonuclear cell & $6.4-8.2 \mathrm{~g} / \mathrm{dL}$ & 1.8 & 1.4 \\
\hline Total protein & $<40$ & Not performed & 50 \\
\hline ADA test & & &
\end{tabular}

Pleural and ascitic fluid analyses of the patient showed chylothorax and chylous ascites. Chylothorax could be related to pulmonary TB that had been diagnosed through sputum smear examination. Meanwhile, the patient's chylous ascites was concluded to be related to extrapulmonary TB in the presence of a positive ADA examination.

\section{Other Diagnostic Examinations}

Other diagnostic examinations for TB are tissue biopsy, nucleic acid amplification by polymerase chain reaction (PCR) method, and immunological test such as Interferon Gamma Releasing Assay (IGRA) (Al-Busafi et al., 2014; Lee, 2015; Arend et al., 2007).

PCR can use a variety of specimens including effusion fluid and shows high specificity and sensitivity, fast, and noninvasive. However, PCR test for TB diagnosis is expensive and it requires skilled personnel and lot of equipments
(Afrasiabian et al., 2013; Tseng et al., 2014). PCR tests also cannot differentiate living bacilli from dead bacilli. Thus, these tests continue to give positive results even after successful treatment. The PCR tests are positive in $95 \%$ to $100 \%$ of culture positive cases and in $50 \%$ to $60 \%$ of culture negative cases (Kim et al., 2011).

IGRA, a T-cell assay relies on the stimulation of host blood cells with $M$. tuberculosis-specific antigens and measure the production of interferon gamma. The T-cell assays have proven to be more specific than the TST but are currently unable to distinguish between active disease and latent tuberculosis infection. Therefore, interpretation of the results remains dependent on the clinical context. The costs and technical demands of IGRA will most likely limit their wider use in resource-poor setting, where better tests are the most needed (Arend et al., 2007).

Tissue biopsy to diagnose peritoneal TB was not performed to this patient because it was an invasive examination. Nucleic acid amplification test of the sputum showed positive M. tuberculosis without rifampicin resistancy. IGRA was not performed because sputum smear examination and ADA test of ascitic fluid had already found to be positive for TB. Finally, we conclude that chylothorax and chylous ascites in this patient were related to TB infection.

\section{Management of Chylous Ascites}

Ideal treatments for patients with combined occurrence of chylotorax and chylous ascites are still challenging. Therapy for the underlying causes of chylous effusion is the most important thing. Therapeutic thoracentesis is the initial step in large chylothoraces that cause respiratory distress. Intercostal tube drainage is the preferred method of thoracentesis in most centers (Nair et al., 2007). In patients with symptomatic ascites, a therapeutic paracentesis should be performed to relieve symptoms and could be repeated as needed. Unless the patient has cirrhosis, the replacement of albumin to prevent post-paracentesis circulatory dysfunction is not recommended (Al-Busafi et al., 2014).

Fasting, together with total parenteral nutrition, can decrease the lymph flow in thoracic duct dramatically from 220 $\mathrm{mL} /$ (kg.hour) to $1 \mathrm{~mL} /(\mathrm{kg}$. hour). Furthermore, total parenteral nutrition restores nutritional deficits and balances metabolic impairments imposed by chylous ascites and repeat sessions of paracentesis (Yang et al., 2013). As long-chain triglyceride (LCT) intake through the gastrointestinal tract will cause an increase in chylous leakage, nutrition support should consist of a low-fat diet supplemented with mediumchain triglyceride (MCT), an enteral nutrition plan with MCT, or total parenteral nutrition plan. Indeed, since MCT and total parenteral nutrition, as two nutrition support methods, can effectively prevent LCT from being absorbed by the gastrointestinal tract, they have been used to reduce chylous leakage early (Pan et al., 2016). MCTs are the diet of choice since they are absorbed directly into the intestinal 
cells and transported as fatty acids and glycerol to the liver via the portal system, while the LCTs need to be converted to monoglycerides and free fatty acids in order to be transported as chylomicrons by the lymph ducts. Monitoring of serum electrolytes, serum albumin, total proteins, and body weight is recommended (Cueto-Aguilera et al., 2016).

Somatostatin and its analog, octreotide, have been used in conservative therapy of chylothorax and chylous ascites. Event though the exact mechanism of somatosatin in the treatment of chylous effusion is not well known, somatostatin reduces the amount of lymph circulation in the major lymphatic vessels, suppresses lymph excretion via somatostatin receptors in the wall of the intestine, and may contribute to a reduction in the production of lymph fluid. Somatostatin and octreotide inhibit the secretion of some pituitary and gastrointestinal hormones, increase splanchnic arteriolar resistance, and consequently reduce gastrointestinal flow and the lymph flow. The use of somatostatin is recommended in the early stages before any invasive treatment starts because it has effects on fistula closure. Somatostatin must be administered intravenously due to its short half-life of 1-3 minutes. Octreotide has a longer halflife of 2 hours and the advantage of subcutaneous administration. Somatostatin is given by continuous infusion pump at a dose of $6 \mathrm{mg}$ a day. Octreotide is given by subcutaneous injection at a dose of $0.1 \mathrm{mg}$ three times a day (Lizaola et al., 2017; Pan et al., 2016; Lee et al., 2014).

Hypoalbuminemia is commonly caused by malnutrition, liver and kidney diseases. Hypoalbuminemia might have resulted from nutritional deficiency or from sepsis. The mechanisms of hypoalbuminemia in patients with sepsis are reduced liver synthesis, accelerated catabolism and increased leakage into the interstitium due to enhanced vascular permeability (Lee et al., 2014).

Surgical management should be adopted if medical management fails. The clinical parameters that prompt surgical intervention are the daily chylous fluid leak exceed $1.5 \mathrm{~L} /$ day in an adult or more than $1 \mathrm{~L} /$ day for a period more than 5 days. Lymphangiography will help to deliniate the anatomy of the lymphatic channel and thoracic duct as well as the site of leak. If the cylous fluid leak can be identified, direct ligation with non-absorbable suture should be performed on either side of the leak (Nair et al., 2007).

This patient was performed thoracocentesis and chest tube insertion to reduce the complaints of dyspnea due to fluidopneumothorax which was later obtained as chylothorax. After chylous ascites was established through proof puncture of ascites, patient was fasted and administered somatostatin pump infusion for 6 days. Anti-TB drugs were adminstered after the diagnosis of TB had been established through sputum smear examination and ADA test of fluid chylous ascites.
During treatment for 11 days, the production of chylothorax gradually decreased and stomach complaints had improved, so it was decided that surgical management was not needed.

\section{Prognosis}

In the past, the mortality due to chylothorax was in excess of $50 \%$. Currently, the morbidity and mortality have improved due to the more aggressive management strategies adopted (Nair et al., 2007). Patients of chylous effusion due to TB infection usually have a very good response to the administration of anti-TB drugs and supportive treatment (Barman et al., 2015).

\section{CONCLUSION}

It had been reported a 22-year-old male patient with complaints of dyspnea, fever, and abdominal distension. Chest X-ray showed fluidopneumothorax and pleuropneumonia. Chylothorax was obtained from the thoracentesis, and chylous ascites was obtained from paracentesis. M. tuberculosis was found on sputum smear examination. Abdominal CT scan showed ascites, bilateral pleural effusion, and lymph node enlargement in the left and right inguinal paraaortas. ADA test was performed on ascitic fluid and positive result was obtained. Chylothorax and chylous ascites in this patient were concluded to be associated with TB infection. Patient was then treated with somatostatin pump infusion and anti-TB drugs. Chylothorax and chylous ascites improved after treatment with somatostatin for 1 week. Anti-TB drugs were planned to be continued up to 9 months.

\section{REFERENCES}

Afrasiabian S, Mohsenpour B, Bagheri KH, Sigari N, Aftabi K. 2013. Diagnostic value of serum adenosine deaminase level in pulmonary tuberculosis. J Res Med Sci 18:252-254.

Al-Busafi SA, Ghali P, Deschenes M, Wong P. 2014. Chylous ascites: evaluation and management. ISRN Hepatol: $1-10$.

Arend SM, Thijsen SF, Leyten EM, Bouwman JJ, Franken WP, et al. 2007. Comparison of two interferon-gamma assays and tuberculin skin test for tracing tuberculosis contacts. Am J Respir Crit Care Med 175: 618-627.

Barman B, Ete T, Warjir SB, Beyong T, Lynrah KG, Nobin H, Singh V. 2015. Chylous ascites and tuberculosis: a case report with literature review. Case Study and Case Rep 5(2): 74-80.

Cueto-Aguilera AD, Ibarra-Sifuentes HR, Delgado-Garcia G, Atilano-Diaz A, Galarza-Delgado DA. 2016. Chylothorax and chylous ascites due to mycobacterium tuberculosis in 
an AIDS patient whose PCR tested negative. Pneumologia; 65(3):161-163.

Dean RK, Subedi R, Karkee A. 2018. Chylous Ascites as a Complication of Intraabdominal Mycobacterium avium Complex Immune Reconstitution Inflammatory Syndrome. Proc (Baylor Univ Med Cent) 31(3):326-327.

Karapolat S, Sanli A, Onen A. 2008. Chylothorax due to Tuberculosis Lymphadenopathy: Report of a Case. Surg Today; 38: 938-41.

Kim JH, Kim YJ, Ki CS, Kim JY, Lee NY. 2011. Evaluation of cobas TaqMan MTB PCR for detection of mycobacterium tuberculosis. J Clin Microbiol 49:173-176.

Kim KJ, Park DW, Choi WS. 2014. Simultaneous chylothorax and chylous ascites due to tuberculosis. Infect Chemoter 46(1):50-53.

Kumar P, Chandra K. 2013. Chylous ascites due to tuberculosis: a case report and review of literature. J Indian Clin Pract 24(7):640-645.

Lee JY. 2015. Diagnosis and treatment of extrapulmonary tuberculosis. Tuberc Respir Dis 78:47-55.

Lee YD, Lee YH, Choi HS. 2014. Chylous ascites in a patient with sepsis caused by bilateral pneumonia. Korean $\mathbf{J}$ Crit Care Med 29(3): 217-221.

Lizaola B, Bonder A, Trivedi HD, Tapper EB, Cardenas A. 2017. Review article: the diagnostic approach and current management of chylous ascites. Aliment Pharmacol Ther 1-9.

Nair SK, Petko M, Hayward MP. 2007. Aetiology and management of chylothorax in adults. Eur J Cardio-thoracic Surg 32: 362-369.

Ninghot A, Mohod K, Kumar S. 2017. Evaluation of serum adenosine deaminase (ADA) values for detection of pulmonary and extra-pulmonary tuberculosis. Int $\mathrm{J}$ Clin Biochem and Research 4(2):106-110.

Pan W, Cai SY, Luo HL, Ouyang SR, Zhang WD, Wei ZR, Wang DL. 2016. The application of nutrition support in conservative treatment of chylous ascites after abdominal surgery. Therapeutics and Clinical Risk Management 12: 607-612.

Shaik IH, Gonzalez-Ibarra F, Khan R, Shah S, Syed AK, Lintz D. 2014. Case report: chylous ascites in a patient with HIV/AIDS: a late complication of mycobacterium avium complex-immune reconstitution inflammatory syndrome. Case Rep in Infect Dis:1-3.

Steinemann DC, Dindo D, Clavien PA, Nocito A. 2011. Atraumatic chylous ascites: systematic review on symptoms and causes. J Am Coll Surg 212(5): 899-909.

Stevanovic G, Pelemis M, Pavlovic M, Lavadinovic L, Dakic Z, Milosevic I, Milosevic B. 2011. Significance of adenosine deaminase serum concentrations in the diagnosis of extrapulmonary tuberculosis. J IMAB-Ann Proceeding 17(1): 130-134.

Tchaou M, Darre T, Mossi KE, Sonhaye L, Djibril M, et al. 2016. Extra-pulmonary tuberculosis: retrospective review of 83 confirmed cases, observed in radiology in Lomé (Togo). Open J of Radiol 6:49-55.

Tseng CC, Huang RM, Chen KT. 2014. Tuberculosis arthritis: epidemiology, diagnosis, treatment. Clin Res Foot Ankle 2(2):1-7.

Wolf DC. 2018. Chylous ascites. Gastroenterol 1:1-10.

Yang C, Zhang J, Wang S, Li C, Kong X, Zhao Z. 2013. Successful management of chylous ascites with total parenteral nutrition and octreotide in children. Nutr Hosp; 28: 2124-2127. 